JOURNAL OF

FUNCTION SPACES AND APPLICATIONS

Volume 4, Number 3 (2006), 243-259
(C) 2006, Scientific Horizon http://www.jfsa.net

\title{
Regularity results for singular elliptic problems
}

\section{Loredana Caso}

(Communicated by Miroslav Englis )

2000 Mathematics Subject Classification. 35J25, 35B65, 35R05.

Keywords and phrases. Elliptic equations, regularity of solutions, discontinuous coefficients and data.

Abstract. Some local and global regularity results for solutions of linear elliptic equations in weighted spaces are proved. Here the leading coefficients are VMO functions, while the hypotheses on the other coefficients and the boundary conditions involve a suitable weight function.

\section{Introduction}

Consider the second order linear differential equation

$$
L_{o} u=-\sum_{i, j=1}^{n} a_{i j} u_{x_{i} x_{j}}=f \quad \text { a. e. in } \Omega
$$

where $L_{o}$ is a uniformly elliptic operator in a bounded open subset $\Omega$ of $\mathbb{R}^{n}, n \geq 3$, and $f \in L^{p}(\Omega), p>1$. A classical problem in the theory of linear elliptic equations in non-divergence form is the study of local and global regularity properties of solutions of (1.1). 
It is well known that, when the $a_{i j}$ 's are uniformly continuous in $\Omega$, any solution $u$ of (1.1) in $W_{\text {loc }}^{2, q}(\Omega), 1<q \leq p$, belongs to $W_{\text {loc }}^{2, p}(\Omega)$ (see [11], Chapter 9). On the other hand, if the coefficients $a_{i j}$ are required to be discontinuous, some other kind of assumptions are necessary to get regularity results for the solutions of (1.1). For instance, if either the $a_{i j}$ 's belong to $W^{1, n}(\Omega)$ or they satisfy the "Cordes condition", then local $W^{2, p}$ regularity results (with $p$ belonging to a suitable neighborhood of $p=2$ ) for the solutions of (1.1) have been obtained (see [10] and [2]).

More recently, some local regularity results in $W^{2, p}$, for any $\left.p \in\right] 1,+\infty[$, have been proved under the assumption that the coefficients $a_{i j}$ are bounded and of class $V M O$ (see [8]). Observe that this latter condition is always satisfied if the $a_{i j}$ 's either are uniformly continuous or lie in $W^{1, n}$. The results of [8] have been extended to the case of operators whose lower order terms occur and belong to suitable spaces $L^{r}(\Omega)$ (see [16]).

If $\Omega$ is an arbitrary open subset of $\mathbb{R}^{n}, n \geq 3$, a $W^{2, p}(\Omega)$-regularity

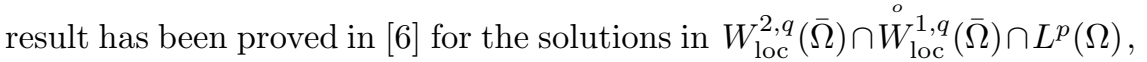
$1<q \leq p$, of the linear elliptic equation

$$
L u=-\sum_{i, j=1}^{n} a_{i j} u_{x_{i} x_{j}}+\sum_{i=1}^{n} a_{i} u_{x_{i}}+a u=f,
$$

$f \in L^{p}(\Omega)$, under the conditions that the coefficients $a_{i j}$ are bounded and belong to the space $\operatorname{VMO}(\Omega)$, while the coefficients $a_{i}$ and $a$ lie in suitable spaces of Morrey type. Moreover, in [4] this result has been improved, obtaining local and global regularity results under weaker assumptions; in particular, the $a_{i j}$ 's are assumed locally $V M O$ and satisfying a suitable condition at infinity.

The aim of this paper is to obtain some local and global regularity results for the solutions of the equation (1.2) in certain weighted Sobolev spaces. More precisely, let $\rho$ be a suitable weight function and denote by $S_{\rho}$ the subset of $\partial \Omega$ where $\rho$ goes to zero. Suppose that there exist extensions $a_{i j}^{o}$ of $a_{i j}$ in $\operatorname{VMO}\left(\Omega_{o}\right) \cap L^{\infty}\left(\Omega_{o}\right)$, where $\Omega_{o}$ is a regular open set containing $\Omega$; assume also that the coefficients $a_{i}$ and $a$ satisfy certain local summability hypotheses and are singular near $S_{\rho}$. If $f \in L_{\text {loc }}^{p}(\Omega)$, we will prove that

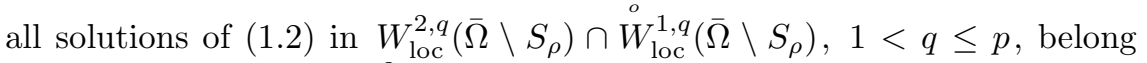
to the Sobolev space $W_{\text {loc }}^{2, p}\left(\bar{\Omega} \backslash S_{\rho}\right)$. Moreover, if $f$ and the solutions of (1.2) belong to certain weighted $L^{p}$-spaces, where the weight functions are suitable powers of $\rho$, then such solutions belong to the weighted Sobolev space $W_{s}^{2, p}(\Omega)$. 


\section{Notation and function spaces}

In this paper we use the following basic notation: $E$, a generic Lebesgue measurable subset of $\mathbb{R}^{n} ; \Sigma(E)$, the Lebesgue $\sigma$-algebra on $E ;|A|$, the Lebesgue measure of $A \in \Sigma(E) ; \chi_{A}$, the caratteristic function of $A ; \mathfrak{D}(A)$, the class of restrictions to $A$ of functions $\zeta \in C_{o}^{\infty}\left(\mathbb{R}^{n}\right)$ with $\bar{A} \cap \operatorname{supp} \zeta \subseteq A$; $L_{\mathrm{loc}}^{p}(A)(p \in[1,+\infty])$, the class of functions $g$, defined on $A$, such that $\zeta g \in L^{p}(A)$ for all $\zeta \in \mathfrak{D}(A) ; B(x, r)$, the open ball of radius $r$ centered at $x$.

Let $\Omega$ be an open subset of $\mathbb{R}^{n}$. For each $E \in \Sigma(\Omega)$ we put

$$
E(x, r)=E \cap B(x, r) \quad \forall x \in \mathbb{R}^{n}, \quad \forall r \in \mathbb{R}_{+} .
$$

Denote by $\mathcal{A}(\Omega)$ the class of measurable functions $\rho: \Omega \rightarrow \mathbb{R}_{+}$such that

$$
\gamma^{-1} \rho(y) \leq \rho(x) \leq \gamma \rho(y) \quad \forall y \in \Omega, \quad \forall x \in \Omega(y, \rho(y))
$$

where $\gamma \in \mathbb{R}_{+}$is independent of $x$ and $y$. For $\rho \in \mathcal{A}(\Omega)$, we put

$$
S_{\rho}=\left\{z \in \partial \Omega: \lim _{x \rightarrow z} \rho(x)=0\right\} .
$$

It is known that

$$
\rho \in L_{\mathrm{loc}}^{\infty}(\bar{\Omega}), \quad \rho^{-1} \in L_{\mathrm{loc}}^{\infty}\left(\bar{\Omega} \backslash S_{\rho}\right),
$$

and, if $S_{\rho} \neq \emptyset$,

$$
\rho(x) \leq \operatorname{dist}\left(x, S_{\rho}\right) \quad \forall x \in \Omega
$$

(see [5], [14]).

If $r \in \mathbb{N}, 1 \leq p \leq+\infty, s \in \mathbb{R}$ and $\rho \in \mathcal{A}(\Omega)$, we consider the space $W_{s}^{r, p}(\Omega)$ of distributions $u$ on $\Omega$ such that $\rho^{s+|\alpha|-r} \partial^{\alpha} u \in L^{p}(\Omega)$ for $|\alpha| \leq r$, equipped with the norm

$$
\|u\|_{W_{s}^{r, p}(\Omega)}=\sum_{|\alpha| \leq r}\left\|\rho^{s+|\alpha|-r} \partial^{\alpha} u\right\|_{L^{p}(\Omega)} .
$$

Moreover, we denote by $\stackrel{o}{W}_{s}^{r, p}(\Omega)$ the closure of $C_{o}^{\infty}(\Omega)$ in $W_{s}^{r, p}(\Omega)$ and put $W_{s}^{0, p}(\Omega)=L_{s}^{p}(\Omega)$. A more detailed account of properties of the above defined spaces can be found in [9], [1] and [15]. 
For $p \in\left[1,+\infty\left[\right.\right.$, denote by $K_{s}^{p}(\Omega)$ the set of all functions $g \in L_{\text {loc }}^{p}\left(\bar{\Omega} \backslash S_{\rho}\right)$ such that

$$
\|g\|_{K_{s}^{p}(\Omega)}=\sup _{x \in \Omega}\left(\rho^{s-n / p}(x)\|g\|_{L^{p}(\Omega(x, \rho(x)))}\right)<+\infty,
$$

endowed with the norm defined by (2.4). Moreover, $\tilde{K}_{s}^{p}(\Omega)$ denotes the closure of $L_{s}^{\infty}(\Omega)$ in $K_{s}^{p}(\Omega)$. It is known that a function $g$ (in $K_{s}^{p}(\Omega)$ ) belongs to $\tilde{K}_{s}^{p}(\Omega)$ if and only if the function

$$
\sigma_{p, g}^{s}(t)=\sup _{\substack{E \in \Sigma(\Omega) \\ \sup _{x \in \Omega} \frac{|E(x, \rho(x))|}{\rho^{n}(x)} \leq t}}\left\|g \chi_{E}\right\|_{K_{s}^{p}(\Omega)}, \quad t \in \mathbb{R}_{+},
$$

vanishes when $t$ goes to zero. Thus a modulus of continuity of $g$ in $\tilde{K}_{s}^{p}(\Omega)$ is a map $\tilde{\sigma}_{p}[g]: \mathbb{R}_{+} \rightarrow \mathbb{R}_{+}$such that

$$
\sigma_{p, g}^{s}(t) \leq \tilde{\sigma}_{p}[g](t) \quad \forall t \in \mathbb{R}_{+}, \quad \lim _{t \rightarrow 0^{+}} \tilde{\sigma}_{p}[g](t)=0 .
$$

For some properties of the spaces $K_{s}^{p}(\Omega)$ and $\tilde{K}_{s}^{p}(\Omega)$ we refer to [3] and [5].

If $\Omega$ has the property

$$
\left.\left.|\Omega(x, r)| \geq A r^{n} \quad \forall x \in \Omega, \quad \forall r \in\right] 0,1\right],
$$

where $A$ is a positive constant independent of $x$ and $r$, it is possible to consider the space $B M O(\Omega, t)\left(t \in \mathbb{R}_{+}\right)$of functions $g \in L_{\text {loc }}^{1}(\bar{\Omega})$ such that

$$
[g]_{B M O(\Omega, t)}=\sup _{\substack{x \in \Omega \\ r \in[0, t]}} f_{\Omega(x, r)}\left|g-f_{\Omega(x, r)} g\right| d y<+\infty,
$$

where $f_{\Omega(x, r)} g d y=\frac{1}{|\Omega(x, r)|} \int_{\Omega(x, r)} g d y$.

If $g \in B M O(\Omega)=B M O\left(\Omega, t_{A}\right)$, where

$$
t_{A}=\sup _{t \in \mathbb{R}_{+}}\left(\sup _{\substack{x \in \Omega \\ r \in j 0, t]}} \frac{r^{n}}{|\Omega(x, r)|} \leq \frac{1}{A}\right),
$$

we shall say that $g \in V M O(\Omega)$ if $[g]_{B M O(\Omega, t)} \rightarrow 0$ for $t \rightarrow 0^{+}$. A function $\eta[g]: \mathbb{R}_{+} \rightarrow \mathbb{R}_{+}$is called a modulus of continuity of $g$ in $V M O(\Omega)$ if

$$
[g]_{B M O(\Omega, t)} \leq \eta[g](t) \quad \forall t \in \mathbb{R}_{+}, \quad \lim _{t \rightarrow 0^{+}} \eta[g](t)=0 .
$$




\section{Preliminary results}

Fix $\rho \in \mathcal{A}(\Omega)$. For all $x \in \Omega$ and for all $\lambda \in \mathbb{R}_{+}$put

$$
\begin{aligned}
E_{\lambda}(x) & =\{y \in \Omega:|x-y|<\lambda \rho(y)\}, & & E(x)=E_{1}(x), \\
I_{\lambda}(x) & =\Omega \cap B(x, \lambda \rho(x)), & & I(x)=I_{1}(x) .
\end{aligned}
$$

Obviously

$$
y \in I_{\lambda}(x) \Leftrightarrow x \in E_{\lambda}(y) \quad \forall x, y \in \Omega .
$$

It is easy to prove that

$$
E(x) \subset I_{\gamma}(x) \quad \forall x \in \Omega,
$$

and

$$
I_{\lambda_{o} \gamma^{-1}}(x) \subset E_{\lambda}(x) \quad \forall x \in \Omega,
$$

for all $\lambda \in \mathbb{R}_{+}$, where $\gamma$ is the constant in (2.1) and $\lambda_{o}=\min \{\lambda, \gamma\}$.

We consider the following conditions on $\rho$ :

$(\alpha)$ there exists an extension $\tilde{\rho} \in \mathcal{A}\left(\mathbb{R}^{n} \backslash S_{\rho}\right)$ of $\rho$ to $\mathbb{R}^{n} \backslash S_{\rho}$,

( $\beta) \quad H=\inf _{\Omega} \rho^{-n}(x)|I(x)| \in \mathbb{R}_{+}$.

Remark 3.1. If condition $(\alpha)$ holds and $S_{\rho} \neq \emptyset$, then

$$
S_{\tilde{\rho}}=S_{\rho}
$$

and there exists $a \in] 0,1[$ such that

$$
\rho(x) \leq a \operatorname{dist}\left(x, S_{\rho}\right) \quad \forall x \in \Omega
$$

(see [3] and [5]).

If $(\alpha)$ holds, we consider the sets $\tilde{E}_{\lambda}(x)$ and $\tilde{I}_{\lambda}(x), x \in \mathbb{R}^{n} \backslash S_{\rho}$, defined by (3.1), (3.2), respectively, corresponding to $\rho=\tilde{\rho}$. From Remark 3.1 it follows that

$$
\left.\left.\tilde{I}_{\lambda}(x)=B(x, \lambda \tilde{\rho}(x)) \quad \forall x \in \mathbb{R}^{n} \backslash S_{\rho}, \quad \forall \lambda \in\right] 0,1\right] .
$$

Lemma 3.1. If condition $(\alpha)$ holds, then

$$
\omega_{n} \gamma^{-n} \tilde{\rho}^{n}(x) \leq|\tilde{E}(x)| \leq \omega_{n} \gamma^{n} \tilde{\rho}^{n}(x) \quad \forall x \in \mathbb{R}^{n} \backslash S_{\rho},
$$


while if $(\beta)$ holds, then

$$
H \rho^{n}(x) \leq|E(x)| \leq \omega_{n} \gamma^{n} \rho^{n}(x) \quad \forall x \in \Omega,
$$

where $\omega_{n}$ is the volume of the unit ball in $\mathbb{R}^{n}$.

Proof. The result easily follows from (3.4), (3.5) and (3.8).

Now we define the function $\rho_{o}: \mathbb{R}^{n} \backslash S_{\rho} \rightarrow \mathbb{R}_{+}$by

$$
\begin{aligned}
& \rho_{o}(x)=\tilde{\rho}(x) \\
& \rho_{o}(x)= \begin{cases}\rho(x) & \text { if }(\alpha) \text { holds }, \\
0 & x \in \mathbb{R}^{n} \backslash\left(\Omega \cup S_{\rho}\right) .\end{cases}
\end{aligned}
$$

For any $x \in \mathbb{R}^{n} \backslash S_{\rho}$, put

$$
\begin{aligned}
I^{o}(x) & =\left(\mathbb{R}^{n} \backslash S_{\rho}\right) \cap B\left(x, \rho_{o}(x)\right), \\
E^{o}(x) & =\left\{y \in \mathbb{R}^{n} \backslash S_{\rho}:|y-x|<\rho_{o}(y)\right\},
\end{aligned}
$$

and for $x, y \in \mathbb{R}^{n} \backslash S_{\rho}$, let

$$
\Phi^{o}(x, y)= \begin{cases}1 & y \in I^{o}(x) \\ 0 & y \notin I^{o}(x) .\end{cases}
$$

Lemma 3.2. Assume that either $(\alpha)$ or $(\beta)$ holds. Then for any $p, q \in\left[1,+\infty\left[, q \geq p\right.\right.$, and for all $s \in \mathbb{R}$ there exist $c_{1}, c_{2} \in \mathbb{R}_{+}$such that

$$
\begin{aligned}
& \int_{\mathbb{R}^{n} \backslash S_{\rho}} \rho_{o}^{s p-n}(x)\left\|u_{o}\right\|_{L^{p}\left(I^{o}(x)\right)}^{p} d x \geq c_{1} \int_{\Omega} \rho^{s p}(x)|u(x)|^{p} d x \\
& \int_{\mathbb{R}^{n} \backslash S_{\rho}} \rho_{o}^{s q-n}(x)\left\|u_{o}\right\|_{L^{p}\left(I^{o}(x)\right)}^{q} d x \leq c_{2}\left(\int_{\Omega} \rho^{s p}(x)|u(x)|^{p} d x\right)^{\frac{q}{p}}
\end{aligned}
$$

for all $u \in L_{\text {loc }}^{p}\left(\bar{\Omega} \backslash S_{\rho}\right)$, where $u_{o}$ is the zero extension of $u$ outside $\Omega$, $c_{1}$ depends on $\gamma, n, s, p$ and also on $H$ if $(\beta)$ holds, while $c_{2}$ depends on $\gamma, n, s, p, q$.

Proof. Fix $p \in\left[1,+\infty\left[, s \in \mathbb{R}\right.\right.$ and $u \in L_{\text {loc }}^{p}\left(\bar{\Omega} \backslash S_{\rho}\right)$. Then

$$
\begin{aligned}
& \int_{\mathbb{R}^{n} \backslash S_{\rho}} \rho_{o}^{s p-n}(x)\left\|u_{o}\right\|_{L^{p}\left(I^{o}(x)\right)}^{p} d x \\
& \quad=\int_{\mathbb{R}^{n} \backslash S_{\rho}}\left(\rho_{o}^{s p-n}(x) \int_{I^{o}(x)}\left|u_{o}(y)\right|^{p} \Phi^{o}(x, y) d y\right) d x
\end{aligned}
$$




$$
=\int_{\mathbb{R}^{n} \backslash S_{\rho}}\left(\left|u_{o}(y)\right|^{p} \int_{\mathbb{R}^{n} \backslash S_{\rho}} \rho_{o}^{s p-n}(x) \Phi^{o}(x, y) d x\right) d y .
$$

So by (2.1), (3.3) and Lemma 3.1, we deduce (3.11).

Fix now $q \geq p$ and put

$$
h(x)=\rho_{o}^{s(q-p)}(x)\left(\int_{I^{o}(x)}\left|u_{o}(y)\right|^{p} d y\right)^{q / p-1}, \quad x \in \mathbb{R}^{n} \backslash S_{\rho} .
$$

Thus from (2.1) we have

$$
\begin{aligned}
\int_{\mathbb{R}^{n} \backslash S_{\rho}} \rho_{o}^{s q-n}(x)\left\|u_{o}\right\|_{L^{p}\left(I^{o}(x)\right)}^{q} d x \\
=\int_{\mathbb{R}^{n} \backslash S_{\rho}}\left(\rho_{o}^{s p-n}(x) h(x) \int_{I^{o}(x)}\left|u_{o}(y)\right|^{p} \Phi^{o}(x, y) d y\right) d x \\
\leq c_{3} \int_{\mathbb{R}^{n} \backslash S_{\rho}}\left(\rho_{o}^{\left(s-\frac{n}{q}\right) p}(y)\left|u_{o}(y)\right|^{p}\left(\int_{E^{o}(y)} d x\right)^{p / q}\right) d y \\
\cdot\left(\int_{\mathbb{R}^{n} \backslash S_{\rho}} \rho_{o}^{s q-n}(x)\left\|u_{o}\right\|_{L^{p}\left(I^{o}(x)\right)}^{q} d x\right)^{1-p / q},
\end{aligned}
$$

where $c_{3}$ depends on $\gamma, s, p, q$. It follows from Lemma 3.1 that (3.12) holds.

Consider the following hypothesis on $\Omega$ :

$\left(i_{1}\right)$ there exist $\left.\left.b \in\right] 0,1\right]$ and $\left.\theta \in\right] 0, \frac{\pi}{2}[$ such that

$$
\forall x \in \Omega \quad \exists C_{\theta}(x): \overline{C_{\theta}(x, b \rho(x))} \subset \Omega,
$$

where $C_{\theta}(x)$ is an open indefinite cone with vertex in $x$ and opening $\theta$ and $C_{\theta}(x, b \rho(x))=C_{\theta}(x) \cap B(x, b \rho(x))$.

Remark 3.2. If condition $\left(i_{1}\right)$ holds, then also $(\beta)$ holds (see [14]).

Fix $k, r \in \mathbb{N}, p, p_{o} \in[1,+\infty$ [ and consider the following condition

(i $\left.) \frac{1}{r p}+\frac{r-1}{p_{o}} \leq 1,0 \leq k<r, \frac{k}{r} \leq a \leq \vartheta, \vartheta \in\right] \frac{r-1}{r}, 1\left[, q \geq \max \left\{p, p_{o}\right\}\right.$

where $\frac{1}{q}=\frac{k}{n}+a\left(\frac{1}{p}-\frac{r}{n}\right)+\frac{1-a}{p_{o}}$.

Lemma 3.3. Assume that conditions $\left(i_{1}\right)$ and $\left(i_{2}\right)$ hold. Let $\alpha_{1}, \alpha_{2} \in$ $\mathbb{R}$ such that

$$
\alpha_{1}-\alpha_{2}=r+\frac{n}{p_{o}}-\frac{n}{p}
$$


Then for any function $u$ such that $\partial^{r} u \in L_{\mathrm{loc}}^{p}(\Omega), \partial^{r} u \in L_{\alpha_{1}}^{p}(\Omega), u \in$ $L_{\alpha_{2}}^{p_{o}}(\Omega)$, we have

$$
\left\|\partial^{k} u\right\|_{L_{\alpha_{o}}^{q}(\Omega)} \leq c\left(\left\|\partial^{r} u\right\|_{L_{\alpha_{1}}^{p}(\Omega)}^{a} \cdot\|u\|_{L_{\alpha_{2}}^{p o}(\Omega)}^{1-a}+\|u\|_{L_{\alpha_{2}}^{p_{o}(\Omega)}}\right),
$$

where $\alpha_{o}=a \alpha_{1}+(1-a) \alpha_{2}$ and $c \in \mathbb{R}_{+}$depends on $\Omega, n, r, k, \alpha_{1}, \alpha_{2}, p, p_{o}, q, \rho$.

Proof. The proof is similar to that of Theorem 2.1 in [13], using Lemma 3.2 instead of Lemmas 1.1 and 1.2 of [13].

We recall now two imbedding results proved in [5] that will be useful for our pourposes. Consider real numbers $r, p, q, s$ such that

(i, $) \quad r \in \mathbb{N}, s \in \mathbb{R}, 1 \leq p \leq q<+\infty, q \geq \frac{n}{r}, q>\frac{n}{r}$ if $\frac{n}{r}=p>1$.

Lemma 3.4. Suppose that conditions $\left(i_{1}\right)$ and $\left(i_{3}\right)$ hold. Then for all $g \in K_{-s+r}^{q}(\Omega)$ and for all $u \in W_{s}^{r, p}(\Omega)$, we have $g u \in L^{p}(\Omega)$ and

$$
\|g u\|_{L^{p}(\Omega)} \leq c_{o}\|g\|_{K_{-s+r}^{q}(\Omega)}\|u\|_{W_{s}^{r, p}(\Omega)},
$$

where $c_{o}$ depends on $\Omega, n, p, q, r, s, \rho$.

Lemma 3.5. Suppose that the hypotheses of Lemma 3.4 are satisfied and let $g \in \tilde{K}_{-s+r}^{q}(\Omega)$. Then for any $\varepsilon \in \mathbb{R}_{+}$there exists $c(\varepsilon) \in \mathbb{R}_{+}$such that

$$
\|g u\|_{L^{p}(\Omega)} \leq \varepsilon\|u\|_{W_{s}^{r, p}(\Omega)}+c(\varepsilon)\|u\|_{L_{s-r}^{p}(\Omega)},
$$

where $c(\varepsilon)$ depends on $\varepsilon, \Omega, n, p, q, r, s, \rho,\|g\|_{K_{-s+r}^{q}(\Omega)}$ and $\tilde{\sigma}_{q}[g]$.

Observe that in the original statements of the above lemmas given in [5], the dependence of the constants was not explicited.

\section{An a priori bound}

From now on we suppose that $n \geq 3$. Fix $\rho \in \mathcal{A}(\Omega) \cap L^{\infty}(\Omega)$ and consider the following condition on $\Omega$ :

$\left(h_{1}\right)$ there exists an open subset $\Omega_{o}$ of $\mathbb{R}^{n}$ with the uniform $C^{1,1}$ regularity property, such that

$$
\Omega \subset \Omega_{o}, \quad \partial \Omega \backslash S_{\rho} \subset \partial \Omega_{o} .
$$

Remark 4.1. If condition $\left(h_{1}\right)$ holds and $\rho \in \mathcal{A}(\Omega) \cap L^{\infty}(\Omega)$, then $\Omega$ satisfies $\left(i_{1}\right)$ (see [5]). 
Let $p \in] 1,+\infty[, s \in \mathbb{R}$. Consider in $\Omega$ the differential operator

$$
L=-\sum_{i, j=1}^{n} a_{i j} \frac{\partial^{2}}{\partial x_{i} \partial x_{j}}+\sum_{i=1}^{n} a_{i} \frac{\partial}{\partial x_{i}}+a
$$

with the following conditions on the coefficients:

$\left(h_{2}\right)$ there exist extensions $a_{i j}^{o}$ of $a_{i j}$ to $\Omega_{o}$ such that

$$
\begin{aligned}
&\left\{\begin{array}{l}
a_{i j}^{o}=a_{j i}^{o} \in L^{\infty}\left(\Omega_{o}\right) \cap V M O\left(\Omega_{o}\right), \quad i, j=1, \ldots n, \\
\exists \nu \in \mathbb{R}_{+}: \sum_{i, j=1}^{n} a_{i j}^{o} \xi_{i} \xi_{j} \geq \nu|\xi|^{2} \quad \text { a.e. in } \Omega_{o}, \quad \forall \xi \in \mathbb{R}^{n},
\end{array}\right. \\
&\left(h_{3}\right) \quad\left\{\begin{array}{ll}
a_{i} \in \tilde{K}_{1}^{t_{1}}(\Omega), & i=1, \ldots, n, \text { where } t_{1} \geq p, \quad t_{1} \geq n, \\
a \in \tilde{K}_{2}^{t_{2}}(\Omega), & \text { and } t_{1}>p \text { if } p=n,
\end{array} \quad \text { and } t_{2}>p \text { if } p=\frac{n}{2} .\right.
\end{aligned}
$$

We put

$$
L_{o}=-\sum_{i, j=1}^{n} a_{i j} \frac{\partial^{2}}{\partial x_{i} \partial x_{j}} .
$$

Theorem 4.1. Assume that conditions $\left(h_{1}\right)-\left(h_{3}\right)$ hold. Then there exists a positive real number $c$ such that

$(4.1)\|u\|_{W_{s}^{2, p}(\Omega)} \leq c\left(\|L u\|_{L_{s}^{p}(\Omega)}+\|u\|_{L_{s-2}^{p}(\Omega)}\right) \forall u \in W_{s}^{2, p}(\Omega) \cap \stackrel{o}{W_{s-1}^{1, p}(\Omega),}$

where $c$ depends on $\Omega, n, p, t_{1}, t_{2}, s, \rho, \nu,\left\|a_{i j}^{o}\right\|_{L^{\infty}\left(\Omega_{o}\right)},\left\|a_{i}\right\|_{K_{1}^{t_{1}}(\Omega)},\|a\|_{K_{2}^{t_{2}(\Omega)}}$, $\eta\left[a_{i j}^{o}\right], \tilde{\sigma}_{t_{1}}\left[a_{i}\right]$ and $\tilde{\sigma}_{t_{2}}[a]$.

Proof. Fix $u \in W_{s}^{2, p}(\Omega) \cap \stackrel{o}{W_{s-1}^{1, p}}(\Omega)$. Consider a function $\phi \in C_{o}^{\infty}\left(\mathbb{R}^{n}\right)$ such that

$$
0 \leq \phi \leq 1, \quad \phi_{\left.\right|_{B\left(0, \frac{1}{2}\right)}}=1, \quad \operatorname{supp} \phi \subset B(0,1) .
$$

For each $x \in \Omega$, let

$$
\psi=\psi^{x}: y \in \mathbb{R} \rightarrow \phi\left(\frac{y-x}{\rho(x)}\right) ;
$$

then

$$
\left\{\begin{array}{l}
0 \leq \psi \leq 1, \quad \psi_{\left.\right|_{\frac{1}{2}(x)}}=1, \quad \operatorname{supp} \psi \cap \Omega \subset I(x), \\
\left|\partial^{\alpha} \psi\right| \leq c_{\alpha} \rho^{-|\alpha|}(x) \quad \forall \alpha \in \mathbb{N}_{o}^{n} .
\end{array}\right.
$$


If $u_{o}$ denotes the zero extension of $u$ outside $\Omega$, it follows from (2.2), (2.3) and (3.2) that

$$
\psi u_{o} \in W^{2, p}\left(\Omega_{o}\right) \cap \stackrel{o}{W^{1, p}}\left(\Omega_{o}\right) .
$$

We consider in $\Omega_{o}$ the differential operator

$$
\mathfrak{L}_{o}=-\sum_{i, j=1}^{n} a_{i j}^{o} \frac{\partial^{2}}{\partial x_{i} \partial x_{j}} .
$$

Then from hypotheses $\left(h_{1}\right),\left(h_{2}\right)$ and from Theorem 5.1 in [6], we have

(4.5) $\left\|\left(\psi u_{o}\right)_{x x}\right\|_{L^{p}\left(\Omega_{o}\right)} \leq c_{1}\left(\left\|\mathfrak{L}_{o}\left(\psi u_{o}\right)\right\|_{L^{p}\left(\Omega_{o}\right)}+\left\|\psi u_{o}\right\|_{L^{p}\left(\Omega_{o}\right)}\right)$,

where $c_{1}$ depends on $\Omega, n, p, \nu,\left\|a_{i j}^{o}\right\|_{L^{\infty}\left(\Omega_{o}\right)}, \eta\left[a_{i j}^{o}\right]$.

Observe that

$$
\left\|\mathfrak{L}_{o}\left(\psi u_{o}\right)\right\|_{L^{p}\left(\Omega_{o}\right)}=\left\|L_{o}(\psi u)\right\|_{L^{p}(\Omega)}
$$

and

$$
L_{o}(\psi u)=\psi L_{o} u-\sum_{i, j=1}^{n} a_{i j} \psi_{x_{i} x_{j}} u-2 \sum_{i, j=1}^{n} a_{i j} \psi_{x_{i}} u_{x_{j}} .
$$

Thus, from (4.6) and (4.7) we deduce

$$
\begin{aligned}
& \left\|\mathfrak{L}_{o}\left(\psi u_{o}\right)\right\|_{L^{p}\left(\Omega_{o}\right)}+\left\|\psi u_{o}\right\|_{L^{p}\left(\Omega_{o}\right)} \\
\leq & c_{2}\left(\left\|\psi L_{o} u\right\|_{L^{p}(\Omega)}+\left\|\psi_{x x} u\right\|_{L^{p}(\Omega)}+\left\|\psi_{x} u_{x}\right\|_{L^{p}(\Omega)}+\|\psi u\|_{L^{p}(\Omega)}\right),
\end{aligned}
$$

where $c_{2}$ depends on $n, p,\left\|a_{i j}^{o}\right\|_{L^{\infty}\left(\Omega_{o}\right)}$.

It follows from (4.4), (4.5) and (4.8) that

$$
\begin{aligned}
& \rho^{-2}(x)\|u\|_{L^{p}\left(I_{\frac{1}{2}}(x)\right)}+\rho^{-1}(x)\left\|u_{x}\right\|_{L^{p}\left(I_{\frac{1}{2}}(x)\right)}+\left\|u_{x x}\right\|_{L^{p}\left(I_{\frac{1}{2}}(x)\right)} \\
& \leq c_{3}\left(\left\|L_{o} u\right\|_{L^{p}(I(x))}+\rho^{-2}(x)\|u\|_{L^{p}(I(x))}+\rho^{-1}(x)\left\|u_{x}\right\|_{L^{p}(I(x))}\right),
\end{aligned}
$$

where $c_{3}$ depends on $\Omega, n, p, \rho, \nu,\left\|a_{i j}^{o}\right\|_{L^{\infty}\left(\Omega_{o}\right)}, \eta\left[a_{i j}^{o}\right]$. 
Then by (4.9) and by Lemma 3.2 we have

$$
\begin{gathered}
\int_{\Omega} \rho^{(s-2) p}(x)|u|^{p} d x+\int_{\Omega} \rho^{(s-1) p}(x)\left|u_{x}\right|^{p} d x+\int_{\Omega} \rho^{s p}(x)\left|u_{x x}\right|^{p} d x \\
\leq c_{4}\left(\int_{\Omega} \rho^{s p}(x)\left|L_{o} u\right|^{p} d x+\int_{\Omega} \rho^{(s-2) p}(x)|u|^{p} d x\right. \\
\left.+\int_{\Omega} \rho^{(s-1) p}(x)\left|u_{x}\right|^{p} d x\right)
\end{gathered}
$$

where $c_{4}$ depends on the same parameters as $c_{3}$.

Therefore

$$
\|u\|_{W_{s}^{2, p}(\Omega)} \leq c_{5}\left(\left\|L_{o} u\right\|_{L_{s}^{p}(\Omega)}+\|u\|_{L_{s-2}^{p}(\Omega)}+\left\|u_{x}\right\|_{L_{s-1}^{p}(\Omega)}\right),
$$

where $c_{5}$ depends on the same parameters as $c_{4}$.

Moreover, by $\left(h_{3}\right)$ and Lemma 3.5, for each $\varepsilon \in \mathbb{R}_{+}$there exists $c(\varepsilon) \in \mathbb{R}_{+}$ such that

$$
\begin{aligned}
& \left\|\sum_{i=1}^{n} a_{i} u_{x_{i}}+a u\right\|_{L_{s}^{p}(\Omega)} \\
& \quad \leq \varepsilon\|u\|_{W_{s}^{2, p}(\Omega)}+c(\varepsilon)\left(\|u\|_{L_{s-2}^{p}(\Omega)}+\left\|u_{x}\right\|_{L_{s-1}^{p}(\Omega)}\right),
\end{aligned}
$$

where $c(\varepsilon)$ depends on $\varepsilon, \Omega, n, p, t_{1}, t_{2},, s, \rho,\left\|a_{i}\right\|_{K_{1}^{t_{1}}(\Omega)},\|a\|_{K_{2}^{t_{2}(\Omega)}}, \tilde{\sigma}_{t_{1}}\left[a_{i}\right]$ and $\tilde{\sigma}_{t_{2}}[a]$.

Using (4.11) and (4.12), we have

$$
\|u\|_{W_{s}^{2, p}(\Omega)} \leq c_{6}\left(\|L u\|_{L_{s}^{p}(\Omega)}+\|u\|_{L_{s-2}^{p}(\Omega)}+\left\|u_{x}\right\|_{L_{s-1}^{p}(\Omega)}\right)
$$

with $c_{6} \in \mathbb{R}_{+}$depending on $\Omega, n, p, t_{1}, t_{2}, s, \rho, \nu,\left\|a_{i j}^{o}\right\|_{L^{\infty}\left(\Omega_{o}\right)}$, $\left\|a_{i}\right\|_{K_{1}^{t_{1}}(\Omega)},\|a\|_{K_{2}^{t_{2}(\Omega)}}, \eta\left[a_{i j}^{o}\right], \tilde{\sigma}_{t_{1}}\left[a_{i}\right]$ and $\tilde{\sigma}_{t_{2}}[a]$.

On the other hand, from Lemma 3.3 there exists $c_{7} \in \mathbb{R}_{+}$, depending on $\Omega, n, p, s, \rho$, such that

$$
\left\|u_{x}\right\|_{L_{s-1}^{p}(\Omega)} \leq c_{7}\left(\left\|u_{x x}\right\|_{L_{s}^{p}(\Omega)}^{\frac{1}{2}} \cdot\|u\|_{L_{s-2}^{p}(\Omega)}^{\frac{1}{2}}+\|u\|_{L_{s-2}^{p}(\Omega)}\right) .
$$

Finally, (4.1) follows from (4.13) and (4.14). 


\section{Main results}

In this section we will prove two regularity results, the first of local and the other of global type. In the case of local regularity, we require that the coefficients of lower order satisfy suitable local summability conditions:

$$
\left(h^{\prime}{ }_{3}\right) \quad\left\{\begin{array}{l}
a_{i} \in L_{\text {loc }}^{t_{1}}\left(\bar{\Omega} \backslash S_{\rho}\right), i=1, \ldots, n, \text { where } t_{1} \geq p, \quad t_{1}>n, \\
a \in L_{\text {loc }}^{t_{2}}\left(\bar{\Omega} \backslash S_{\rho}\right), \text { where } t_{2} \geq p, \quad t_{2}>\frac{n}{2} .
\end{array}\right.
$$

For $p \in] 1,+\infty\left[\right.$, we denote by $W_{\text {loc }}^{2, p}\left(\bar{\Omega} \backslash S_{\rho}\right)$ and $\stackrel{\circ}{W_{\text {loc }}^{1, p}}\left(\bar{\Omega} \backslash S_{\rho}\right)$ the spaces of all functions $u: \Omega \rightarrow \mathbb{R}$ such that $\zeta u \in W^{2, p}(\Omega)$ or $\zeta u \in \stackrel{o}{W}^{1, p}(\Omega)$, respectively, for each $\zeta \in \mathfrak{D}\left(\bar{\Omega} \backslash S_{\rho}\right)$.

Theorem 5.1. Assume that conditions $\left(h_{1}\right),\left(h_{2}\right)$ and $\left(h^{\prime}{ }_{3}\right)$ hold and let $u$ be a solution of the problem

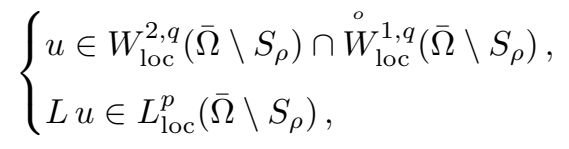

where $q \in] 1, p]$. Then $u \in W_{\text {loc }}^{2, p}\left(\bar{\Omega} \backslash S_{\rho}\right)$.

Proof. Assume that $q<p$ and fix $\psi \in \mathfrak{D}\left(\bar{\Omega} \backslash S_{\rho}\right)$. We claim that $\psi u \in W^{2, p}(\Omega)$. To this aim, let $k \in \mathbb{N}$ such that

$$
k-1<n\left(\frac{1}{q}-\frac{1}{p}\right) \leq k,
$$

so that

$$
\frac{1}{q_{h}}=\frac{1}{q}-\frac{h}{n}>\frac{1}{p}, \quad h=0, \cdots, k-1, \quad \frac{1}{q}-\frac{k}{n} \leq \frac{1}{p}=\frac{1}{q_{k}} .
$$

Thus it is enough to prove that

$$
\psi u \in W^{2, q_{h}}(\Omega) \Rightarrow \psi u \in W^{2, q_{h+1}}(\Omega)
$$

for all $h=0, \cdots, k-1$.

Observe that

$$
\psi u_{o} \in W^{2, q_{h}}\left(\Omega_{o}\right) \cap \stackrel{o}{W^{1, q_{h}}}\left(\Omega_{o}\right),
$$


where $u_{o}$ is the zero extension of $u$ outside $\Omega$. Let $A$ be an open subset of $\mathbb{R}^{n}$ such that

$$
\operatorname{supp} \psi \subset A, \quad A \cap \bar{\Omega} \subset \bar{\Omega} \backslash S_{\rho},
$$

and fix $\chi \in C_{o}^{\infty}(A)$ such that

$$
\chi=1 \quad \text { on } \quad \operatorname{supp} \psi .
$$

Consider in $\Omega_{o}$ the differential operator

$$
\mathfrak{L}=-\sum_{i, j=1}^{n} a_{i j}^{o} \frac{\partial^{2}}{\partial x_{i} \partial x_{j}}+\sum_{i=1}^{n} a_{i}^{o} \frac{\partial}{\partial x_{i}}+a^{o},
$$

where $a_{i}^{o}$ and $a^{o}$ are the zero extensions of $a_{i}$ and $a$, respectively, outside $\Omega$. Observe that

$$
\left\|\mathfrak{L}\left(\psi u_{o}\right)\right\|_{L^{q_{h+1}}\left(\Omega_{o}\right)}=\|L(\psi u)\|_{L^{q_{h+1}}(\Omega)}
$$

and

$$
L(\psi u)=\psi L u+\sum_{i=1}^{n} \alpha_{i}(\chi u)_{x_{i}}+\alpha(\chi u)
$$

where

$$
\begin{aligned}
\alpha_{i} & =-2 \sum_{j=1}^{n} a_{i j} \psi_{x_{j}}, \quad i=1, \ldots, n, \\
\alpha & =-\sum_{i, j=1}^{n} a_{i j} \psi_{x_{i} x_{j}}+\sum_{i=1}^{n} a_{i} \psi_{x_{i}} .
\end{aligned}
$$

Using now the same argument of the proof of Lemma 4.1 in [4], it can be proved that $L(\psi u) \in L^{q_{h+1}}(\Omega)$ and so, by (5.5), also that $\mathfrak{L}\left(\psi u_{o}\right) \in$ $L^{q_{h+1}}\left(\Omega_{o}\right)$. Application of Lemma 4.2 of [7] yields that $\psi u_{o} \in W^{2, q_{h+1}}\left(\Omega_{o}\right)$ and hence (5.3) holds.

Theorem 5.2. Suppose that conditions $\left(h_{1}\right)-\left(h_{3}\right)$ hold with $t_{1}>n$ and $t_{2}>n / 2$. Then any solution $u$ of the problem

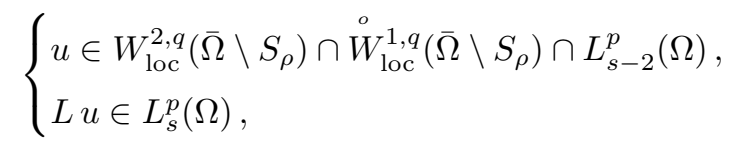

(with $q \in] 1, p]$ ) belongs to $W_{s}^{2, p}(\Omega)$. 
Proof. Fix a solution $u$ of problem (5.7). By Theorem 5.1 we have that $u \in W_{\text {loc }}^{2, p}\left(\bar{\Omega} \backslash S_{\rho}\right)$. Let $\left.r, r^{\prime} \in\right] 0,1\left[\right.$ with $r<r^{\prime}$ and $\phi \in C_{o}^{\infty}\left(\mathbb{R}^{n}\right)$ such that

$$
\phi_{\left.\right|_{B(0, r)}}=1, \quad \operatorname{supp} \phi \subset B\left(0, r^{\prime}\right), \quad \sup _{\mathbb{R}^{n}}\left|\partial^{\alpha} \phi\right| \leq c_{\alpha}\left(r^{\prime}-r\right)^{-|\alpha|} \quad \forall \alpha \in \mathbb{N}_{o}^{n} .
$$

For each $x \in \Omega$, consider the function $\psi$ defined by (4.3). In our situation we obtain

$$
\left\{\begin{array}{l}
\psi_{\left.\right|_{B(x, r \rho(x))}}=1, \quad \operatorname{supp} \psi \subset B\left(x, r^{\prime} \rho(x)\right), \\
\sup _{\mathbb{R}^{n}}\left|\partial^{\alpha} \psi\right| \leq c_{\alpha}\left(r^{\prime}-r\right)^{-|\alpha|} \rho^{-|\alpha|}(x) \quad \forall \alpha \in \mathbb{N}_{o}^{n} .
\end{array}\right.
$$

It is easy to prove that $\psi u \in W_{s}^{2, p}(\Omega) \cap \stackrel{o}{W_{s-1}^{1, p}}(\Omega)$ and so it follows from Theorem 4.1 that

$$
\|\psi u\|_{W_{s}^{2, p}(\Omega)} \leq c_{1}\left(\|L(\psi u)\|_{L_{s}^{p}(\Omega)}+\|\psi u\|_{L_{s-2}^{p}(\Omega)}\right),
$$

where $c_{1}$ depends on $\Omega, n, p, t_{1}, t_{2}, s, \rho, \nu,\left\|a_{i j}^{o}\right\|_{L^{\infty}\left(\Omega_{o}\right)},\left\|a_{i}\right\|_{K_{1}^{t_{1}(\Omega)}}$, $\|a\|_{K_{2}^{t_{2}}(\Omega)}, \eta\left[a_{i j}^{o}\right], \tilde{\sigma}_{t_{1}}\left[a_{i}\right]$ and $\tilde{\sigma}_{t_{2}}[a]$.

Note that

$$
L(\psi u)=\psi L u+\sum_{i, j=1}^{n} a_{i j} \psi_{x_{i} x_{j}} u-2 \sum_{i, j=1}^{n} a_{i j}\left(\psi_{x_{i}} u\right)_{x_{j}}+\sum_{i=1}^{n} a_{i} \psi_{x_{i}} u,
$$

and so, by (5.9),

$$
\begin{aligned}
\|\psi u\|_{W_{s}^{2, p}(\Omega)} \leq & c_{2}\left(\|\psi L u\|_{L_{s}^{p}(\Omega)}+\left\|\sum_{i=1}^{n}\left(\psi_{x_{i}} u\right)_{x}\right\|_{L_{s}^{p}(\Omega)}\right. \\
& \left.+\left\|\psi_{x x} u\right\|_{L_{s}^{p}(\Omega)}+\left\|\sum_{i=1}^{n} a_{i} \psi_{x_{i}} u\right\|_{L_{s}^{p}(\Omega)}+\|\psi u\|_{L_{s-2}^{p}(\Omega)}\right)
\end{aligned}
$$

where $c_{2}$ depends on the same parameters as $c_{1}$.

From Lemma 3.3 we have

$$
\begin{aligned}
& \left\|\left(\psi_{x_{i}} u\right)_{x}\right\|_{L_{s}^{p}(\Omega)} \\
& \quad \leq \quad c_{3}\left(\left\|\left(\psi_{x_{i}} u\right)_{x x}\right\|_{L_{s+1}^{p}(\Omega)}^{\frac{1}{2}} \cdot\left\|\psi_{x_{i}} u\right\|_{L_{s-1}^{p}(\Omega)}^{\frac{1}{2}}+\left\|\psi_{x_{i}} u\right\|_{L_{s-1}^{p}(\Omega)}\right),
\end{aligned}
$$

where $c_{3}$ depends on $\Omega, n, p, s, \rho$. 
Moreover, (5.8) gives

$$
\begin{aligned}
\leq & c_{4}\left(\left\|\psi_{x x x} u\right\|_{L_{s+1}^{p}\left(I_{r^{\prime}}(x)\right)}+\left\|\psi_{x x} u_{x}\right\|_{L_{s+1}^{p}\left(I_{r^{\prime}}(x)\right)}+\left\|\psi_{x} u_{x x}\right\|_{L_{s+1}^{p}\left(I_{r^{\prime}}(x)\right)}\right) \\
\leq & c_{5}\left(\left(r^{\prime}-r\right)^{-3} \rho^{-3}(x)\|u\|_{L_{s+1}^{p}\left(I_{r^{\prime}}(x)\right)}+\left(r^{\prime}-r\right)^{-2} \rho^{-2}(x)\left\|u_{x}\right\|_{L_{s+1}^{p}\left(I_{r^{\prime}}(x)\right)}\right. \\
& \left.+\left(r^{\prime}-r\right)^{-1} \rho^{-1}(x)\left\|u_{x x}\right\|_{L_{s+1}^{p}\left(I_{r^{\prime}}(x)\right)}\right)
\end{aligned}
$$

where $c_{4}$ depends on $n, p$, and $c_{5}$ depends on $n, p, \rho$.

Since $\left(r^{\prime}-r\right)^{-1} \leq\left(r^{\prime}-r\right)^{-2}$, it follows from (5.11), (5.12) and (5.8) that

$$
\begin{aligned}
\left\|\left(\psi_{x_{i}} u\right)_{x}\right\|_{L_{s}^{p}(\Omega)} \leq & c_{6}\left(\left(r^{\prime}-r\right)^{-2} \rho^{-2}(x)\|u\|_{L_{s}^{p}\left(I_{r^{\prime}}(x)\right)}+\left(r^{\prime}-r\right)^{-1}\right. \\
& \left.\cdot \rho^{-1}(x)\left\|u_{x}\right\|_{L_{s}^{p}\left(I_{r^{\prime}}(x)\right)}+\left\|u_{x x}\right\|_{L_{s}^{p}\left(I_{r^{\prime}}(x)\right)}\right)^{\frac{1}{2}} \\
& \cdot\left(\left(r^{\prime}-r\right)^{-2} \rho^{-2}(x)\|u\|_{L_{s}^{p}\left(I_{r^{\prime}}(x)\right)}\right)^{\frac{1}{2}}
\end{aligned}
$$

where $c_{6}$ depends on $\Omega, n, p, s, \rho$.

On the other hand, by Lemma 3.4, (5.8) and (5.13) we have

$$
\begin{aligned}
\left\|a_{i} \psi_{x_{i}} u\right\|_{L_{s}^{p}(\Omega) \leq} & c_{7}\left\|\psi_{x_{i}} u\right\|_{W_{s-1}^{2, p}(\Omega)} \\
\leq & c_{8}\left(\rho^{-1}(x)\left\|\psi_{x_{i}} u\right\|_{L_{s}^{p}\left(I_{r^{\prime}}(x)\right)}+\left\|\left(\psi_{x_{i}} u\right)_{x}\right\|_{L_{s}^{p}\left(I_{r^{\prime}}(x)\right)}\right) \\
\leq & c_{9}\left(\left(r^{\prime}-r\right)^{-2} \rho^{-2}(x)\|u\|_{L_{s}^{p}\left(I_{r^{\prime}}(x)\right)}+\left(r^{\prime}-r\right)^{-1}\right. \\
& \left.\cdot \rho^{-1}(x)\left\|u_{x}\right\|_{L_{s}^{p}\left(I_{r^{\prime}}(x)\right)}+\left\|u_{x x}\right\|_{L_{s}^{p}\left(I_{r^{\prime}}(x)\right)}\right)^{\frac{1}{2}} \\
& \cdot\left(\left(r^{\prime}-r\right)^{-2} \rho^{-2}(x)\|u\|_{L_{s}^{p}\left(I_{r^{\prime}}(x)\right)}\right)^{\frac{1}{2}}
\end{aligned}
$$

where $c_{7}-c_{9}$ depend on $\Omega, n, p, t_{1}, s, \rho$ and $\left\|a_{i}\right\|_{K_{1}^{t_{1}}(\Omega)}$.

Therefore by (5.8), (5.10), (5.13) and (5.14) we obtain

$$
\begin{aligned}
& \rho^{-2}(x)\|u\|_{L_{s}^{p}\left(I_{r}(x)\right)}+\rho^{-1}(x)\left\|u_{x}\right\|_{L_{s}^{p}\left(I_{r}(x)\right)}+\left\|u_{x x}\right\|_{L_{s}^{p}\left(I_{r}(x)\right)} \\
& \quad \leq c_{10}\|\psi u\|_{W_{s}^{2, p}(\Omega)}
\end{aligned}
$$




$$
\begin{array}{r}
\leq c_{11}\left(r^{\prime}-r\right)^{-2}\left[\|L u\|_{L_{s}^{p}\left(I_{r^{\prime}}(x)\right)}+\left(\rho^{-2}(x)\|u\|_{L_{s}^{p}\left(I_{r^{\prime}}(x)\right)}+\rho^{-1}(x)\right.\right. \\
\left.\left.\cdot\left\|u_{x}\right\|_{L_{s}^{p}\left(I_{r^{\prime}}(x)\right)}+\left\|u_{x x}\right\|_{L_{s}^{p}\left(I_{r^{\prime}}(x)\right)}\right)^{\frac{1}{2}} \cdot\left(\rho^{-2}(x)\|u\|_{L_{s}^{p}\left(I_{r^{\prime}}(x)\right)}\right)^{\frac{1}{2}}\right],
\end{array}
$$

where $c_{10}$ depends on $p, \rho$, and $c_{11}$ depends on $\Omega, n, p, t_{1}, t_{2}, s, \rho, \nu$, $\left\|a_{i j}^{o}\right\|_{L^{\infty}\left(\Omega_{o}\right)},\left\|a_{i}\right\|_{K_{1}^{t_{1}}(\Omega)},\|a\|_{K_{2}^{t_{2}}(\Omega)}, \eta\left[a_{i j}^{o}\right], \tilde{\sigma}_{t_{1}}\left[a_{i}\right]$ and $\tilde{\sigma}_{t_{2}}[a]$.

Applying a well-known lemma of monotonicity due to C. Miranda (see [12], Lemma 3.1) it follows that

$$
\begin{aligned}
& \rho^{-2}(x)\|u\|_{L_{s}^{p}\left(I_{\frac{1}{2}}(x)\right)}+\rho^{-1}(x)\left\|u_{x}\right\|_{L_{s}^{p}\left(I_{\frac{1}{2}}(x)\right)}+\left\|u_{x x}\right\|_{L_{s}^{p}\left(I_{\frac{1}{2}}(x)\right)} \\
& \quad \leq \quad c_{12}\left(\|L u\|_{L_{s}^{p}(I(x))}+\rho^{-2}(x)\|u\|_{L_{s}^{p}(I(x))}\right),
\end{aligned}
$$

with $c_{12}$ dependent on the same parameters as $c_{11}$.

By Lemma 3.2 we deduce

$$
\|u\|_{W_{s}^{2, p}(\Omega)} \leq c_{13}\left(\|L u\|_{L_{s}^{p}(\Omega)}+\|u\|_{L_{s-2}^{p}(\Omega)}\right),
$$

where $c_{13}$ depends on the same parameters as $c_{11}$. Finally, (5.17) proves the result.

\section{References}

[1] V. Benci and D. Fortunato, Weighted Sobolev spaces and the nonlinear Dirichlet problem in unbounded domains, Ann. Mat. Pura Appl., 121 (1979), 319-336.

[2] S. Campanato, Un risultato relativo alle equazioni ellittiche del secondo ordine di tipo non variazionale, Ann. Scuola Norm. Sup. Pisa, 21 (1967), 701-707.

[3] A. Canale, L. Caso, and P. Di Gironimo, Weighted norm inequalities on irregular domains, Rend. Accad. Naz. Sci. XL Mem. Mat., 16 (1992), 193-209.

[4] L. Caso, P. Cavaliere and M. Transirico, Uniqueness results for elliptic equations with VMO-coefficients, Int. J. Pure Appl. Math., 13 (2004), 499-512.

[5] L. Caso and M. Transirico, Some remarks on a class of weight functions, Comment. Math. Univ. Carolin., 37 (1996), 469-477. 
[6] P. Cavaliere, M. Longobardi and A. Vitolo, Imbedding estimates and elliptic equations with discontinuous coefficients in unbounded domains, Matematiche (Catania), 51 (1996), 87-104.

[7] P. Cavaliere, M. Transirico and M. Troisi, Uniqueness result for elliptic equations in unbounded domains, Matematiche (Catania), 54 (1999), 139-146.

[8] F. Chiarenza, F. Frasca and P. Longo, Interior $W^{2, p}$ estimates for nondivergence elliptic equations with discontinuous coefficients, Ricerche Mat., 15 (1991), 149-168.

[9] D. E. Edmunds and W. D. Evans, Elliptic and degenerate-elliptic operators in unbounded domains, Ann. Sc. Norm. Sup. di Pisa, 27 (1973), 591-640.

[10] M. Franciosi and N. Fusco, $W^{2, p}$ regularity for the solutions of elliptic non divergence form equations with rough coefficients, Ricerche Mat., 38 (1989), 93-106.

[11] D. Gilbarg and N. S. Trudinger, Elliptic partial differential equations of second order, 2nd edition, Springer-Verlag, Berlin (1983).

[12] C. Miranda, Teoremi di unicità in domini non limitati e teoremi di Liouville per le soluzioni dei problemi al contorno relativi alle equazioni ellittiche, Ann. Mat. Pura Appl., 59 (1962), 189-212.

[13] M. Troisi, Teoremi di inclusione negli spazi di Sobolev con peso, Ricerche Mat., 18 (1969), 49-74.

[14] M. Troisi, Su una classe di funzoni peso, Rend. Accad. Naz. Sci. XL Mem. Mat., 10 (1986), 141-152.

[15] M. Troisi, Su una classe di spazi di Sobolev con peso, Rend. Accad. Naz. Sci. XL Mem. Mat., 10 (1986), 177-189.

[16] C. Vitanza, $W^{2, p}$-regularity for a class of elliptic second order equations with discontinuous coefficients, Matematiche (Catania), 17 (1992), 177-186.

Dipartimento di Matematica e Informatica

Facoltà di Scienze MM.FF.NN.

Università di Salerno

Via Ponte don Melillo

I 84084 Fisciano (SA)

Italy

(E-mail : lorcaso@unisa.it)

(Received : May 2005) 


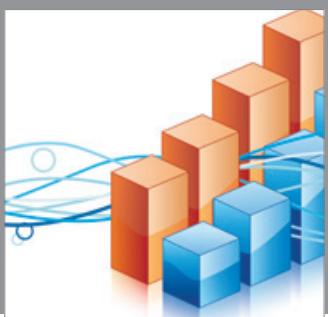

Advances in

Operations Research

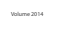

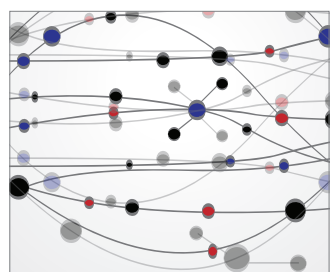

\section{The Scientific} World Journal
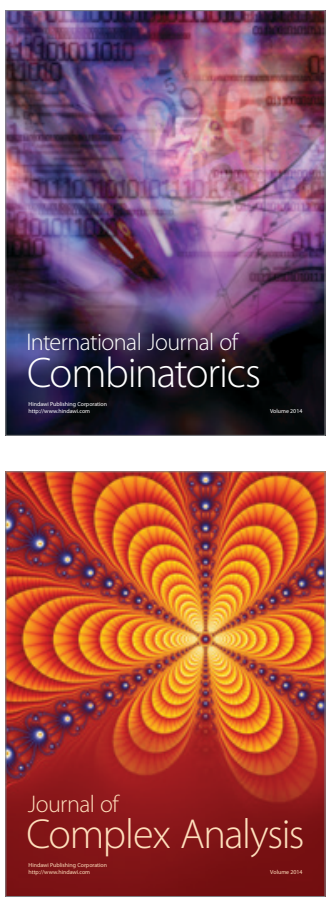

International Journal of

Mathematics and

Mathematical

Sciences
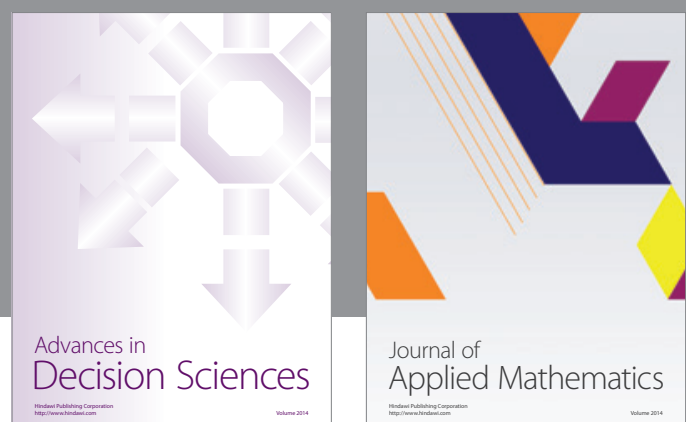

Journal of

Applied Mathematics
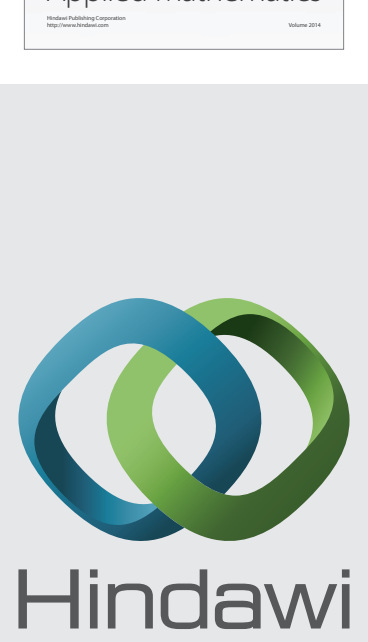

Submit your manuscripts at http://www.hindawi.com
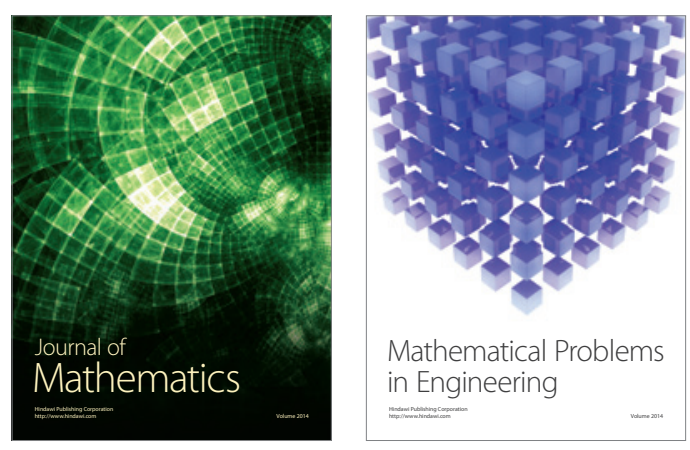

Mathematical Problems in Engineering
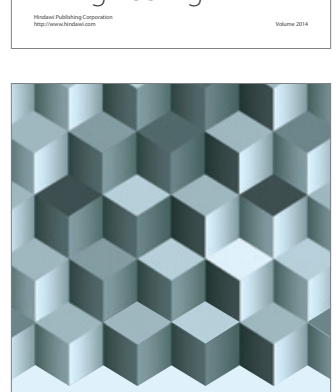

Journal of

Function Spaces
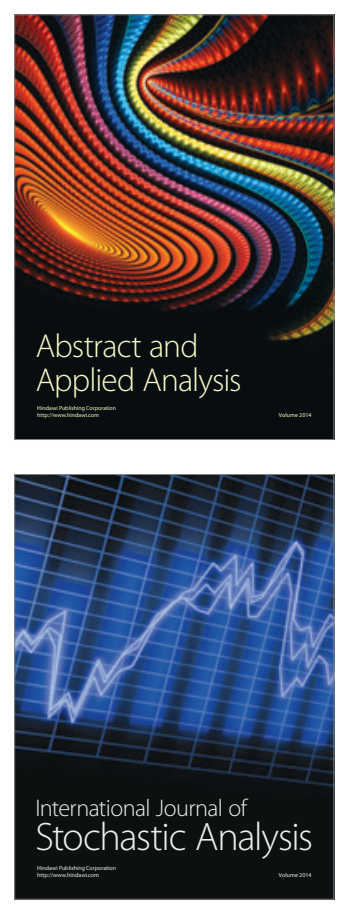

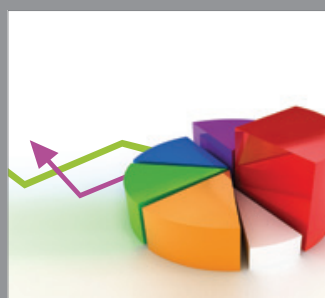

ournal of

Probability and Statistics

Promensencen
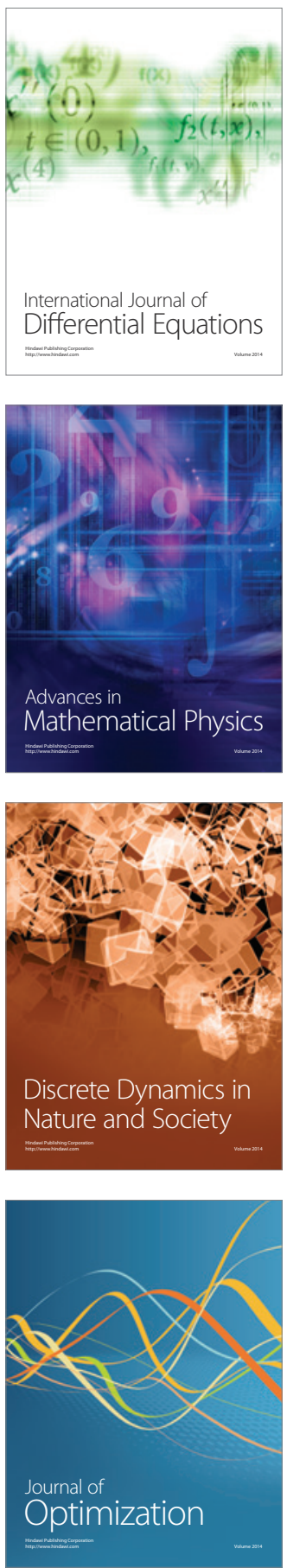